\title{
Comprehensive Analysis Using CT Anthropometry Verifies the Association of Psoas and Masseter Muscle With Traumatic Brain Injury
}

\section{Hang Joo Cho}

Uijeongbu St.Mary's Hospital

\section{Yunsup Hwang}

Uijeongbu St.Mary's Hospital

\section{Sei-Yun Yang}

Uijeongbu St.Mary's Hospital

Maru Kim ( $\square$ maru@catholic.ac.kr)

Department of Trauma Surgery, Uijeongbu St. Mary's Hospital, College of Medicine, The Catholic University of Korea, Seoul, Republic of Korea https://orcid.org/0000-0002-8973-5622

Research article

Keywords: Sarcopenia, Traumatic brain in-jury, Psoas, Masseter muscle, Computed tomography,

Prognosis

Posted Date: September 18th, 2020

DOl: https://doi.org/10.21203/rs.3.rs-76634/v1

License: (c) (1) This work is licensed under a Creative Commons Attribution 4.0 International License.

Read Full License 


\section{Abstract}

Background: Psoas and masseter muscle are known markers for sarcopenia. However, superiority between them is controversial. Authors planned study to analyze them with prognosis of patients with traumatic brain injury. (TBI)

Methods: Patients with TBI visiting regional trauma center between 2017 January to 2018 December were selected and their medical records were reviewed. TBI patients with abbreviated injury score (AIS) 4 or 5 were selected. Patients with AIS 4 or 5 at chest, abdomen and extremity were excluded. And Patient with hospital stay 1 or 2 days were excluded. Both muscle area were measured at their initial computed tomograpy and psoas muscle index (PMI) and masseter muscle index (MMI) were calculated by both muscle area divided by height squared. $(\mathrm{cm} 2 / \mathrm{m} 2)$ These muscular parameters along with other medical information were analyzed with mortality and Glasgow outcome scale. (GOS)

Results: After exclusion process, total 179 patients were included to statistical analyses. Their mean age was 58.0 years, and there are 147 male patients. (82.1\%) There were 30 patients with mortality. (16.8\%) Mean GOS were 3.7 retrospectively. Parameters were analyzed with mortality which is defined as a qualitative outcome. PMA (16.9 vs. $\left.14.4 \mathrm{~cm}^{2}, \mathrm{P}=0.028\right)$ and $\mathrm{PMI}\left(5.9\right.$ vs. $\left.5.1 \mathrm{~cm}^{2} / \mathrm{m}^{2}, \mathrm{P}=0.004\right)$ showed statistical difference. PMI also showed its statistical significance as a risk factor of mortality in logistic regression analysis. $(B=-0.336, P=0.023$, Odds ratio $(95 \%$ confidence interval $)=0.715(0.535-0.954)$ Also analyses was performed with GOS which is defined as a quantitative outcome. Bivariate correlation analysis showed statistical correlation between PMI and GOS. (correlation coefficient $=0.168, P=0.003$ ) And $P M I(B=0.184, P=0.004$, variation inflation factor 1.001$)$ showed its significance in multiple regression analysis additionally.

Conclusions: Bigger PM was associated survival and better GOS statistically and could show its superiority as a prognostic marker. Further analyses with more number of patients, more parameters and more precise setting could yield better outcome and increase understanding of relation between sarcopenia and TBI.

\section{Background}

Sarcopenia is defined as a progressive and generalized skeletal muscle disorder.[1] It was derived from the Greek phrase poverty of flesh. And it works as an important marker of nutritional, physical status and overall health. $[2,3]$ It is now recognized as a muscle disease and considered an independent condition by the International Classification of disease, 10th Clinical Modification.[4, 5] Clinically, it is associated with cardiovascular, respiratory and malignant disease.[6-8] Also individuals' quality of life and mortality show association to sarcopenia.[9, 10]

There are many methods to assess sarcopenia. Measuring psoas muscle area (PMA) from computed tomography (CT) is one of well known method. It is reported that PMA is related to mortality and quality of life of several critical patients.[11-13] Measuring masseter muscle area (MMA) via CT is alternative 
method to analyze sarcopenia. Masseter muscle is associated with nutritional parameters like obesity, geriatrics.[14] Also it shows association with prognosis of patient with cerebrovascular problem.[15, 16] And Hu et al reported that MMA is associated with early mortality of patients with severe traumatic brain injury (TBI).[17]

Both PMA and MMA have enough evidence to prove themselves as valid markers for prognosis of various patients. However, evidence to verify superiority as a prognostic marker between both muscles are not enough. Hereby, authors planned a study to compare both markers with prognosis of patients with TBI. Comprehensive analyses with qualitative and quantitative parameters were performed.

\section{Methods}

Patients with TBI visiting regional trauma center between 2017 January to 2018 December were selected. Their medical information were gathered via retrospective record review. General information like age, sex, height, weight, length of hospital stay and trauma related information like intubation, operation, Glasgow coma score (GCS), abbreviated injury score (AIS), injury severity score (ISS) were checked. As a clinical outcome, Glasgow outcome scale (GOS) and mortality were analyzed together. Patients under endotracheal intubation, GCS 8 or below were regarded as patients with severe TBI. Patients' body mass index were calculated and those with BMI over 25 were regarded as obesity.

Initial CT images were used to muscle measurement. Common sites were used for anthropometry according to previous study. The cross-sectional area of the psoas muscles was measured at the level of the third lumbar vertebral body bilaterally as PMA.[18] The cross-sectional area of the masseter muscle was measured at $2 \mathrm{~cm}$ below the zygomatic arch bilaterally as MMA.[19] Psoas muscle index (PMI) and masseter muscle index (MMI) were calculated by both muscle area divided by height squared. $(\mathrm{cm} 2 / \mathrm{m} 2)$ Patients with proper CT images were included to the study. If their muscle were injured, in tilted position or had artifacts, they were regarded as improper.

To select major TBI, patients with AIS 4 or 5 were extracted from entire patients with TBI. Then, those with improper CT images were excluded. And patients with AIS 4 or 5 at chest, abdomen or extremity were excluded to reduce bias form other condition. Patients younger than 19 years old and those with missing data were excluded also. Additionally, patients with hospital stay less than 2 days were excluded.

Comprehensive analyses were planned. Mortality was used as qualitative outcome and GOS was used as quantitative outcome. At first, patients were separated into survival group and mortality group. Their medical records were analyzed. An independent t-test was used for quantitative analysis, and Chi-squared tests were used for qualitative analysis. And logistic regression analysis was added to verify risk factor. Correlation between both muscular index and GOS was analyzed with bivariate correlation analysis. Correlation coefficient were calculated after adjustment of Age, BMI, AIS, ISS also. Additionally, multiple regression analysis was performed. All statistical analyses were performed using IBM SPSS Statistics for Windows, V 25.0 (IBM Corp., Armonk, NY). This retrospective study was approved by the institutional review board, and the requirement for informed consent was waived. 


\section{Results}

There were 357 TBI patients with AIS 4-5. Among them, patients with proper CT images were 243 and 226 patients without injury of chest, abdomen or extremity AIS 4-5 were selected. And 20 patients were excluded for age, 7 patients were excluded for missing data. Additionally, 20 patients were excluded for missing data. Patient's selection process were summarized at Fig. 1.

After exclusion process, total 179 patients were included to statistical analyses. Their mean age was 58.0 years, and there are 147 male patients. (82.1\%) There were 51 patients with obesity, (28.5\%) 64 patients with severe TBI (35.8\%) and 92 patients after neurosurgery (51.4\%) Mean PMA and MMA were 16.5 and $9.8 \mathrm{~cm}^{2}$. Also Mean PMI and MMI were 5.8 and $3.5 \mathrm{~cm}^{2} / \mathrm{m}^{2}$. There were 30 patients with mortality. (16.8\%) Mean GOS were 3.7 retrospectively. Other basic information is summarized in Table 1. 
Table 1

Basic information of enrolled patients

\section{Age (years)}

Sex

Height (cm)

Weight $(\mathrm{kg})$

Body mass index $\left(\mathrm{kg} / \mathrm{cm}^{2}\right)$

Obesity

Traumatic brain injury

Operation

Psoas muscle area $\left(\mathrm{cm}^{2}\right)$

Masseter muscle area $\left(\mathrm{cm}^{2}\right)$

Psoas muscle index $\left(\mathrm{cm}^{2} / \mathrm{m}^{2}\right)$

Masseter muscle index $\left(\mathrm{cm}^{2} / \mathrm{m}^{2}\right)$

Abbreviated injury score

Head and neck

Face

Chest

Abdomen

Extremity

External

Injury severity score

Glasgow outcome score
$57.95 \pm 16.24$

Female

Male

147 (82.1\%)

$167.59 \pm 8.22$

$65.65 \pm 11.32$

$23.27 \pm 3.03$

No

128 (71.5\%)

Yes

51 (28.5\%)

Non-severe

115 (64.2\%)

Severe

64 (35.8\%)

No

87 (48.6\%)

Yes

92 (51.4\%)

$16.47 \pm 5.60$

$9.76 \pm 2.50$

$5.80 \pm 1.75$

$3.47 \pm 1.75$

$4.54 \pm 5.0$

$0.36 \pm 0.76$

$1.03 \pm 1.35$

$0.25 \pm 0.75$

$0.75 \pm 1.08$

$0.36 \pm 0.54$

$26.59 \pm 6.43$

$3.65 \pm 1.50$

Quantitative parameters are presented as mean \pm standard deviation. Qualitative parameters are presented as number (percent\%). 


\begin{tabular}{|c|c|c|}
\hline Age (years) & & $57.95 \pm 16.24$ \\
\hline 1 & & 30 (16.8\%) \\
\hline 2 & & $9(5.0 \%)$ \\
\hline 3 & & 35 (19.6\%) \\
\hline 4 & & $24(13.4 \%)$ \\
\hline 5 & & $81(45.3 \%)$ \\
\hline \multirow[t]{2}{*}{ Mortality } & No & $149(83.2 \%)$ \\
\hline & Yes & 30 (16.8\%) \\
\hline
\end{tabular}

Parameters were analyzed with mortality which is defined as a qualitative outcome. PMA (16.9 vs. $\left.14.4 \mathrm{~cm}^{2}, \mathrm{P}=0.028\right)$ and $\mathrm{PMI}\left(5.9\right.$ vs. $\left.5.1 \mathrm{~cm}^{2} / \mathrm{m}^{2}, \mathrm{P}=0.004\right)$ showed statistical difference as well as severity of TBI. $(P<0.001)$ Also AIS of face and external were different statistically. Analysis according to mortality is summarized in Table 2. And Table 3 showed result of logistic regression analysis for mortality. Main risk factor for mortality in patients with TBI is severe TBI itself. However, PMI also showed its statistical significance as a risk factor of mortality. $(B=-0.336, P=0.023$, Odds ratio ( $95 \%$ confidence interval $)=0.715(0.535-0.954)$ Mean MMA and MMI were decreased in mortality group however, they could not show its significance. 
Table 2

Analysis according to mortality

\begin{tabular}{|c|c|c|c|c|}
\hline \multicolumn{2}{|l|}{ Parameters } & \multirow{2}{*}{$\begin{array}{l}\text { Survival } \\
\mathrm{N}=149(83.2 \%)\end{array}$} & \multirow{2}{*}{$\begin{array}{l}\text { Mortality } \\
N=30(16.8 \%)\end{array}$} & \multirow[t]{2}{*}{$p$-value } \\
\hline & & & & \\
\hline Age (years) & & $57.23 \pm 16.59$ & $61.53 \pm 14.08$ & 0.186 \\
\hline \multirow[t]{2}{*}{ Sex } & Female & $24(75.0 \%)$ & $8(25.0)$ & 0.168 \\
\hline & Male & $125(85.0 \%)$ & 22 (15.0\%) & \\
\hline \multicolumn{2}{|l|}{ Height $\left(\mathrm{cm}^{2}\right)$} & $167.76 \pm 8.07$ & $166.73 \pm 9.04$ & 0.535 \\
\hline \multicolumn{2}{|l|}{ Weight (kg) } & $65.76 \pm 11.23$ & $65.08 \pm 11.96$ & 0.767 \\
\hline \multicolumn{2}{|c|}{ Body mass index $\left(\mathrm{kg} / \mathrm{m}^{2}\right)$} & $23.27 \pm 3.08$ & $23.27 \pm 2.85$ & 0.999 \\
\hline \multirow[t]{2}{*}{ Obesity } & No & $106(82.8 \%)$ & $22(17.2 \%)$ & 0.808 \\
\hline & Yes & 43 (84.3\%) & $8(15.7 \%)$ & \\
\hline \multirow[t]{2}{*}{ Traumatic brain injury } & Non-severe & 108 (93.9\%) & $7(6.1 \%)$ & $<0.001$ \\
\hline & Severe & $41(64.1 \%)$ & 23 (35.9\%) & \\
\hline \multirow[t]{2}{*}{ Operation } & No & 77 (88.5\%) & 10 (11.5\%) & 0.067 \\
\hline & Yes & 72 (78.3\%) & $20(21.7 \%)$ & \\
\hline \multicolumn{2}{|c|}{ Psoas muscle area $\left(\mathrm{cm}^{2}\right)$} & $16.88 \pm 5.70$ & $14.43 \pm 4.65$ & 0.028 \\
\hline \multicolumn{2}{|c|}{ Masseter muscle area $\left(\mathrm{cm}^{2}\right)$} & $9.79 \pm 2.54$ & $9.59 \pm 2.32$ & 0.694 \\
\hline \multicolumn{2}{|c|}{ Psoas muscle index $\left(\mathrm{cm}^{2} / \mathrm{m}^{2}\right)$} & $5.94 \pm 1.80$ & $5.10 \pm 1.27$ & 0.004 \\
\hline \multicolumn{2}{|c|}{ Masseter muscle index $\left(\mathrm{cm}^{2} / \mathrm{m}^{2}\right)$} & $3.48 \pm 0.87$ & $3.45 \pm 0.80$ & 0.883 \\
\hline \multicolumn{5}{|c|}{ Abbreviated injury score } \\
\hline \multicolumn{2}{|l|}{ Head and Neck } & $4.53 \pm 0.50$ & $4.60 \pm 0.50$ & 0.487 \\
\hline \multicolumn{2}{|l|}{ Face } & $0.40 \pm 0.80$ & $0.17 \pm 0.46$ & 0.03 \\
\hline \multicolumn{2}{|l|}{ Chest } & $1.03 \pm 1.34$ & $1.03 \pm 1.40$ & 0.981 \\
\hline \multicolumn{2}{|l|}{ Abdomen } & $0.23 \pm 0.72$ & $0.33 \pm 0.88$ & 0.513 \\
\hline \multicolumn{2}{|l|}{ Extremity } & $0.77 \pm 1.08$ & $0.70 \pm 1.12$ & 0.765 \\
\hline \multicolumn{2}{|l|}{ External } & $0.40 \pm 0.54$ & $0.17 \pm 0.46$ & 0.02 \\
\hline
\end{tabular}

An independent t-test was used for quantitative analysis, and Chi-squared tests were used for qualitative analysis. 


\begin{tabular}{|lccc|}
\hline Parameters & Survival & Mortality & p - value \\
\hline Injury severity score & $26.52 \pm 6.33$ & $26.90 \pm 7.00$ & 0.771 \\
\hline $\begin{array}{l}\text { An independent t-test was used for quantitative analysis, and } \\
\text { qualitative analysis. }\end{array}$ & & & \\
\hline
\end{tabular}

Table 3

Result of logistic regression analysis for mortality

\begin{tabular}{|llll|}
\hline Parameters & B & $\mathbf{p}$-value & OR $(95 \% \mathrm{Cl})$ \\
\hline Psoas muscle index & -0.336 & 0.023 & $0.715(0.535-0.954)$ \\
\hline Severe Traumatic brain injury & -2.185 & $<0.001$ & $0.112(0.044-0.287)$ \\
\hline OR, odds ratio; $\mathrm{Cl}$, confidence interval & & \\
\hline
\end{tabular}

Also analyses was performed with GOS which is defined as a quantitative outcome. Bivariate correlation analysis showed statistical correlation between PMI and GOS (correlation coefficient $=0.168, P=0.003$, Fig. 2) Partial correlation analyses was performed after adjustment with age, BMI, AIS and ISS. PMI showed its correlation to GOS in every adjustment, however, MMI could not show its correlation statistically. (Table 4) Result of multiple regression analysis was shown at Table $5\left(R^{2}=0.048\right)$. PMI $(B=$ $0.184, P=0.004$, variation inflation factor 1.001 ) showed its significance. Similarly with analyses for mortality, MMI could not prove its significance. 
Table 4

Results of bivariate and partial correlation analysis with Glasgow Coma Scale

\section{Type of analysis}

Psoas

muscle index
Masseter muscle index

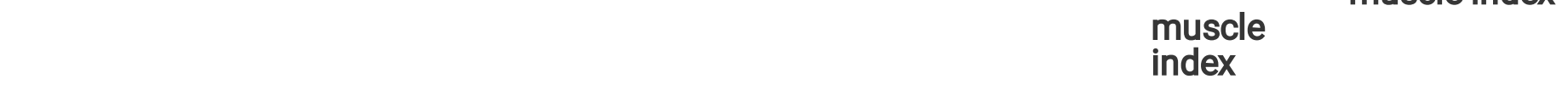

Bivariate correlation Kendall's tau_b analysis

Correlation

0.168

0.061 coefficient

$\mathrm{p}$ - value

0.003

0.281

Partial correlation analysis

\begin{tabular}{llll}
$\begin{array}{l}\text { Adjustment with age, } \\
\text { BMI, AIS, ISS }\end{array}$ & $\begin{array}{l}\text { Correlation } \\
\text { coefficient }\end{array}$ & 0.176 & 0.067 \\
\hline & $p-$ value & 0.023 & 0.390 \\
\hline $\begin{array}{l}\text { Adjustment with BMI, } \\
\text { AIS, ISS }\end{array}$ & $\begin{array}{l}\text { Correlation } \\
\text { coefficient }\end{array}$ & 0.211 & 0.106 \\
\hline & $p-$ value & 0.006 & 0.170 \\
\hline Adjustment with AIS, ISS & $\begin{array}{l}\text { Correlation } \\
\text { coefficient }\end{array}$ & 0.211 & 0.060 \\
\hline & $p-$ value & 0.005 & 0.434 \\
\hline
\end{tabular}

BMI, body mass index; AIS, abbreviated injury score; ISS, injury severity score

Table 5

Result of multiple regression analysis with Glasgow outcome scale

\begin{tabular}{|llll|}
\hline Parameters & B & $\mathbf{p}$-value & Variation Inflation Factor \\
\hline Psoas muscle index & 0.184 & 0.004 & 1.001 \\
\hline AlS - External & 0.476 & 0.02 & 1.001 \\
\hline AlS, abbreviated injury score & & \\
\hline
\end{tabular}

\section{Discussion}

The present study has distinctive characteristics for several reasons. At first, it has a rare purpose of the study. It was planned to compare superiority of PM and SM which were presented as well-known marker for sarcopenia.

Second, the study tried to select severe patients with TBI. Therefore, only TBI patients with AIS 4 or 5 were selected. Also patients with other severe injury were not included to the study. To reduce influence from other injuries, patients with AIS 4 or 5 at chest, abdomen or extremity were excluded. Additionally, patients with hospital stay more than 2 days were selected. Expired trauma patients in this period could have indisputably severe injuries and mortality was non-preventable. On the contrary, there were preventable 
mortality patients who wished "Do Not Resuscitate". Or operation agreement of guardians was late. In addition, there were patients that guardians wanted transfer to other hospital. These patients were regarded as improper candidate for analysis and excluded from the study. Consequently, serious TBI patients with reduced possibility of bias were left, although number of analyzed patients were decreased.

Thirdly, comprehensive analyses were planned with qualitative and and quantitative parameters. There was one similar study that analyzed PM and SM.[20] Both parameters were analyzed with only mortality which is qualitative parameters. However, this study adopted not only mortality, but also GOS and tried to compare both parameters comprehensively.

However, authors could not define and analyze about sarcopenia in the present study. Initial target to analyze was not sarcopenia but both PM and MM. Assessment about muscle strength, physical performance were tried, because enrolled patients had traumatic brain injury and cooperation from the patients was difficult. Also it is reported that single muscle would be difficult to represent sarcopenia.[21] Nevertheless, relation between both muscle and prognosis of patients with TBI could be identified statistically. Further study to diagnose sarcopenia within patients with is required.

\section{Conclusion}

In conclusion, bigger PM was associated survival and better GOS statistically and could show its superiority as a prognostic marker. Although most significant parameter for mortality was severity of TBI itself. Nevertheless, PM showed its statistical significance in various analyses and prove that skeletal muscle could predict prognosis of TBI patients although its correlation coefficients were low. Survival group showed bigger SM also, however it could not prove statistical difference in this study. Further analyses with more number of patients might show different results because this study has relatively low number of participants. Additionally, further with more parameters and more precise setting could yield better outcome and increase understanding of relation between sarcopenia and TBI.

\section{Abbreviations}

PMA

psoas muscle area; CT:computed tomography; MMA:masseter muscle area; TBI:traumatic brain injury; GCS:Glasgow coma score; AIS:abbreviated injury score; ISS:injury severity score; GOS:Glasgow outcome scale; PMI:psoas muscle index; MMI:masseter muscle index

\section{Declarations}

Ethics approval and consent: The present study was approved by Uijeongbu St.Mary's Hospital Institutional Review Board and informed consent was waived.

Consent for publication: Informed consent was waived. 
Availability of data and material: The data generated during the current study are not available because data sharing was not approved from the Institutional Review Board.

Competing interests: Authors have nothing to disclose

Funding: None

Author contribution: HC was involved in study conception, acquisition of data, design, analysis, interpretation. YH was involved in study conception, acquisition of data, interpretation. SY was involved in acquisition of data, interpretation, and validation. MK was involved in study conception, design, analysis, interpretation, and writing of the manuscript. All authors read and approved the final manuscript.

Acknowledgements: None

\section{References}

1. Cruz-Jentoft AJ, Bahat G, Bauer J, Boirie Y, Bruyère O, Cederholm T, et al. Sarcopenia: revised European consensus on definition and diagnosis. Age Ageing. 2019;48:16-31.

2. Cruz-Jentoft AJ, Sayer AA. Sarcopenia. Lancet. 2019;393:2636-46.

3. Gilligan LA, Towbin AJ, Dillman JR, Somasundaram E, Trout AT. Quantification of skeletal muscle mass: sarcopenia as a marker of overall health in children and adults. Pediatr Radiol. 2020;50:45564.

4. Vellas B, Fielding RA, Bens C, Bernabei R, Cawthon PM, Cederholm T, et al. Implications of ICD-10 for Sarcopenia Clinical Practice and Clinical Trials: Report by the International Conference on Frailty and Sarcopenia Research Task Force. J Frailty Aging. 2018;7:2-9.

5. Anker SD, Morley JE, von Haehling S. Welcome to the ICD-10 code for sarcopenia. J Cachexia Sarcopenia Muscle. 2016;7:512-4.

6. Lee SJ, Park YJ, Cartmell KB. Sarcopenia in cancer survivors is associated with increased cardiovascular disease risk. Support Care Cancer. 2018;26:2313-21.

7. Bone AE, Hepgul N, Kon S, Maddocks M. Sarcopenia and frailty in chronic respiratory disease. Chron Respir Dis. 2017;14:85-99.

8. Davis MP, Panikkar R. Sarcopenia associated with chemotherapy and targeted agents for cancer therapy. Ann Palliat Med. 2019;8:86-101.

9. De Buyser SL, Petrovic M, Taes YE, Toye KR, Kaufman JM, Lapauw B, et al. Validation of the FNIH sarcopenia criteria and SOF frailty index as predictors of long-term mortality in ambulatory older men. Age Ageing. 2016;45:602-8.

10. Beaudart C, Biver E, Reginster JY, Rizzoli R, Rolland Y, Bautmans I, et al. Validation of the SarQoL ${ }^{\circledR}$, a specific health-related quality of life questionnaire for Sarcopenia. J Cachexia Sarcopenia Muscle. 2017;8:238-44. 
11. Toptas M, Yalcin M, Akkoc I, Demir E, Metin C, Savas Y, et al. The Relation between Sarcopenia and Mortality in Patients at Intensive Care Unit. Biomed Res Int. 2018;2018:5263208.

12. Drudi LM, Phung K, Ades M, Zuckerman J, Mullie L, Steinmetz OK, et al. Psoas Muscle Area Predicts All-Cause Mortality After Endovascular and Open Aortic Aneurysm Repair. Eur J Vasc Endovasc Surg. 2016;52:764-9.

13. Damluji AA, Rodriguez G, Noel T, Davis L, Dahya V, Tehrani B, et al. Sarcopenia and health-related quality of life in older adults after transcatheter aortic valve replacement. Am Heart J. 2020;224:17181.

14. Hwang Y, Lee YH, Cho DH, Kim M, Lee DS, Cho HJ. Applicability of the masseter muscle as a nutritional biomarker. Medicine. 2020;99:e19069.

15. Oksala NKJ, Lindström I, Khan N, Pihlajaniemi VJ, Lyytikäinen LP, Pienimäki JP, et al. Pre-Operative Masseter Area is an Independent Predictor of Long-Term Survival after Carotid Endarterectomy. Eur $\mathrm{J}$ Vasc Endovasc Surg. 2019;57:331-8.

16. Lindström I, Protto S, Khan N, Hernesniemi J, Sillanpää N, Oksala N. Association of masseter area and radiodensity with three-month survival after proximal anterior circulation occlusion. $\mathrm{J}$ Neurointerv Surg. 2020.

17. Hu P, Uhlich R, White J, Kerby J, Bosarge P. Sarcopenia Measured Using Masseter Area Predicts Early Mortality following Severe Traumatic Brain Injury. J Neurotrauma. 2018;35:2400-6.

18. Hamaguchi Y, Kaido T, Okumura S, Kobayashi A, Hammad A, Tamai Y, et al. Proposal for new diagnostic criteria for low skeletal muscle mass based on computed tomography imaging in Asian adults. Nutrition. 2016;32:1200-5.

19. Tanabe C, Reed MJ, Pham TN, Penn K, Bentov I, Kaplan SJ. Association of Brain Atrophy and Masseter Sarcopenia With 1-Year Mortality in Older Trauma Patients. JAMA Surg. 2019;154:716-23.

20. Wallace JD, Calvo RY, Lewis PR, Brill JB, Shackford SR, Sise MJ, et al. Sarcopenia as a predictor of mortality in elderly blunt trauma patients: Comparing the masseter to the psoas using computed tomography. J Trauma Acute Care Surg. 2017;82:65-72.

21. Baracos VE. Psoas as a sentinel muscle for sarcopenia: a flawed premise. J Cachexia Sarcopenia Muscle. 2017;8:527-8.

\section{Figures}




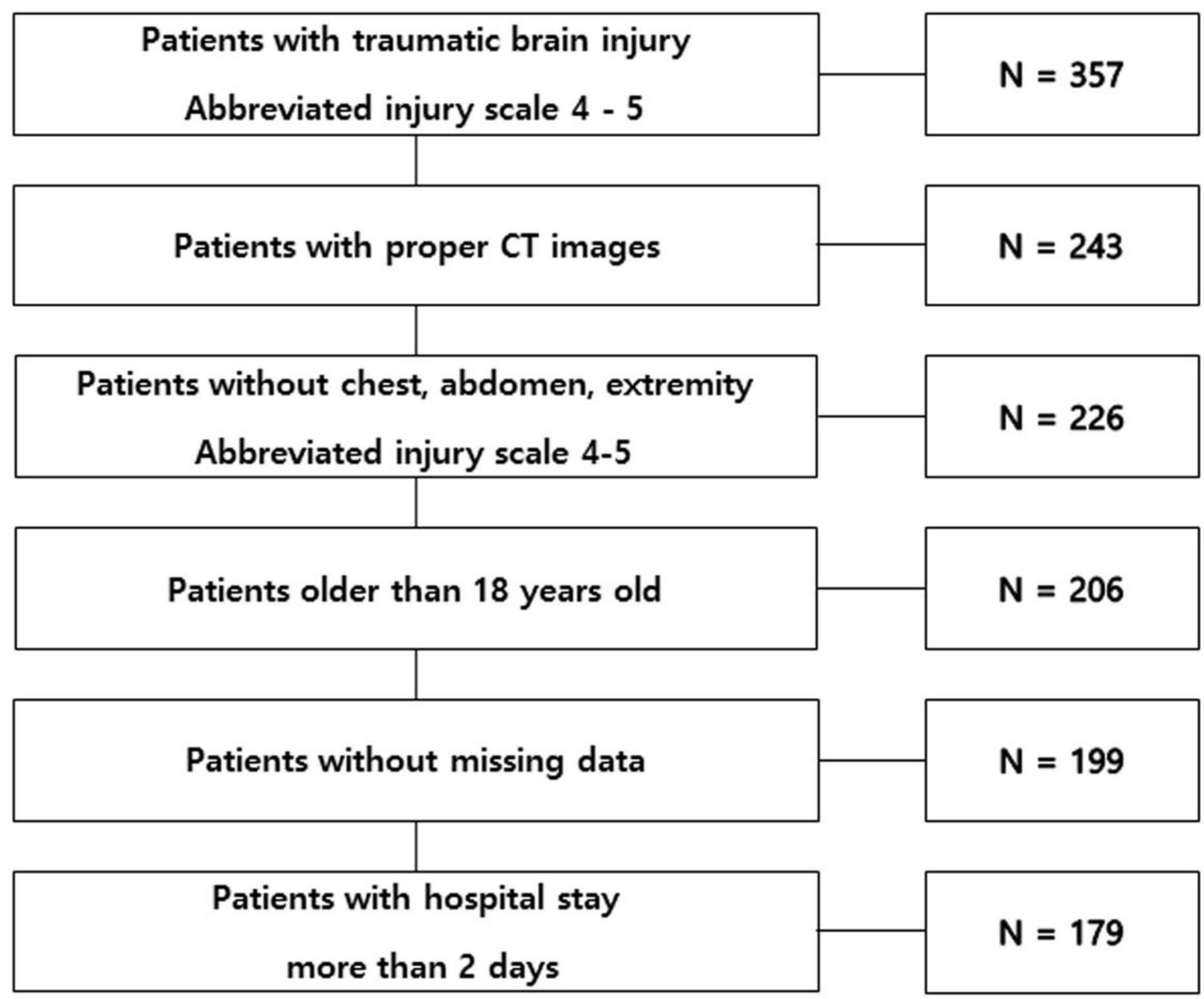

Figure 1

Flowchart showing enrolled patients with traumatic brain injury. 


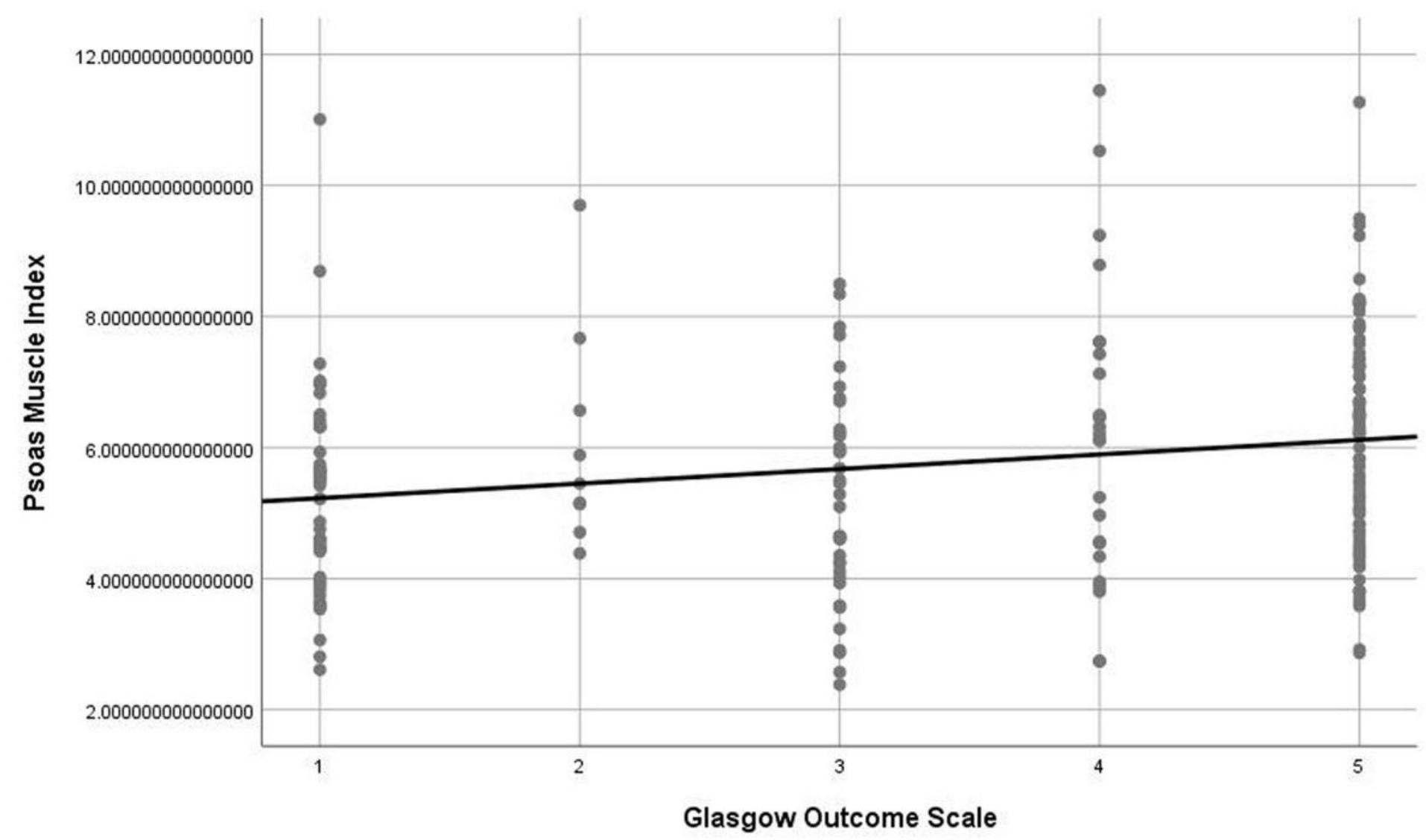

Figure 2

Outcome of correlation between psoas muscle index and Glasgow Outcome Scale. 